\title{
Physical Activity May be a Major Deterrent of Severe Health Consequences from COVID-19: An Annotated Summary of Physical Activity and COVID-19 Research
}

\author{
Marc Bonis* \\ University of New Orleans, Louisiana, USA \\ *Corresponding author: Marc Bonis, University of New Orleans, Louisiana, USA
}

\begin{tabular}{lll}
\hline ARTICLE INFO & ABSTRACT \\
\cline { 1 - 3 } $\begin{array}{l}\text { Received: 幽 July 02, 2020 } \\
\text { Published: 杫 July 17, 2020 }\end{array}$ & $\begin{array}{l}\text { Citation: Marc Bonis. Physical Activity May be a Major Deterrent of Severe Health } \\
\text { Consequences from COVID-19: An Annotated Summary of Physical Activity and } \\
\text { COVID-19 Research. Biomed J Sci \& Tech Res 29(1)-2020. BJSTR. MS.ID.004733. }\end{array}$ \\
\hline
\end{tabular}

\section{Introduction}

During viral or bacterial outbreaks group members with weaker immunological systems are more prone of becoming infected and manifesting virulent effects. Generally, that is the young whose immunological systems have not completely developed and the old whose immunological systems are not as strong as they once were and/or compromised [1]. That is not the case with COVID-19 virus [2]. The young seem to be more resistant and don't seem to be as severely affected as much as the old. Why is that? One of the bigger general differences between the two groups is that the younger group is more physically active than the older group. One has to wonder if physical activity actually reduces the risk of being severely affected. Recent literature regarding physical activity and COVID-19 seems to indicate that this may be the case. If this is true, then voluntary confinement and "sheltering-in-place" strategies may encourage increased sedentary behavior instead of maintaining and/or implementing a major deterrent of the disease. The following eight research articles seem to reinforce the idea that physical activity reduces the risk of health problems due to COVID-19. The first article examines how an extracellular enzyme, extracellular superoxide dismutase (EcSOD), is an anti-oxidant agent, and reduced levels of EcSOD activity is widely present in many chronic disease pathologies. It is widely distributed throughout the body and recent research indicates that endurance exercise increases EcSOD activity. The following excerpts from the article clarifies the recent research:
“Z. Yan, H.Spalding. "Extracellular Superoxide Dismutase, a Molecular Transducer of Health benefits of Exercise." https://doi.org/10.1016/j.redox.2020.101508

Oxygen is indispensable for life; however, under certain situations it has deleterious effects due to the formation and activity of several chemical compounds, known as free radicals or reactive oxygen species (ROS) [3]. Fortunately, cellular enzymatic and nonenzymatic mechanisms are in place to neutralize ROS. Among the members of the enzymatic defense System, Superoxide Dismutases (SODs) are a ubiquitous family of enzymes that catalyze the dismutation of superoxide anions (02.-) as the first line of defense against ROS [4]. However, excessive ROS production can exceed the capacity of the antioxidant defense system leading to oxidative stress, which causes cellular damage by oxidizing proteins, lipids, DNA/RNA, and other macromolecules. Oxidative stress has been shown to play an important role in many disease pathologies, such as cachexia, sepsis, hypertension, Myocardial Infarction (MI), Chronic Heart Failure (CHF), Chronic Obstructive Pulmonary Disease (COPD), Acute Respiratory Distress Syndrome (ARDS) and Multiple Organ Dysfunction Syndrome (MODS) [5].

Extracellular superoxide dismutase (EcSOD) is widely expressed in many tissues/organs with the highest levels in the lung and kidney [6]. EcSOD is so far the only known antioxidant enzyme that functions to scavenge biologically toxic 02.- in the extracellular space. EcSOD is produced within cells, then processed 
and secreted to the extracellular space. The functional importance of EcSOD in humans has been deduced from reduced EcSOD expression in a variety of chronic diseases [7]. Genetic evidence also supports a causal role of reduced EcSOD activity in chronic disease pathologies [8], and it has been associated with increased risk or poor prognosis of oxidative stress-related diseases, such as acute lung injury, ischemic heart diseases, and kidney failure [9]

Overall, EcSOD has recently emerged as a promising therapy for protection of vital tissues/organs under various disease conditions related to oxidative stress. Recent findings suggest that endurance exercise promotes EcSOD expression in skeletal muscle, the largest organ in our body, leading to elevated levels of EcSOD in other peripheral organs. Indeed, controlled clinical trials show that regardless of intensity, volume, type of exercise, and studied population, the antioxidant indicators tend to increase, and prooxidant indicators tend to decrease after exercise training [10] Endurance exercise training has also been shown to increase the abundance of EcSOD in plasma in humans. Importantly, exercise training increases EcSOD protein abundance in peripheral tissues, such as the heart, with no evidence of increased transcription of the EcSOD gene in these peripheral tissues [11]. Exercise-induced EcSOD expression seems to be specific for endurance exercise, not induced by resistance exercise [12]. Enhanced skeletal muscle EcSOD expression by exercise promotes mitigation of oxidative stress and damage in a variety of peripheral tissues and disease conditions.

Accumulating evidence supports that reduced EcSOD abundance and activities in tissues are associated with many disease conditions, and increased EcSOD activity is protective against oxidative stress and damage under these disease pathologies. Endurance exercise increases EcSOD abundance in skeletal tissue, which can be redistributed to peripheral tissues via the circulation to combat ROS and oxidative damage. The facts that EcSOD expression is promoted by endurance exercise in skeletal muscle and can be redistributed to other vital tissues to protect the target tissues against oxidative damage in various pathological processes strongly support exercise-induced EcSOD as an effective therapeutic intervention for prevention and treatment of numerous oxidative stress-related diseases. Enhanced EcSOD through endurance exercise may possibly reduce the risk of acquiring COVID-19." The second article uses VO2max as a risk assessment triage tool and proposes structured physical activity interventions prior to COVID-19 infection. The following excerpt provides additional information about the importance of physical conditioning:

\section{"I. Ahmed. "COVID-19 - Does Exercise Prescription and Maximal Oxygen Uptake (Vo 2 Max) Have a Role in Risk- Stratifying Patients?" (2020) Clin Med. doi: 10.7861/ clinmed.2020-0111.}

As the UK shields 'high risk' patients and enforces social distancing measures, patients will be at risk of significantly reducing physical activity levels. We explore the evidence base for COVID-19-specific recommendations and exercise interventions to 'precondition' patients prior to infection and appraise the role of maximal oxygen uptake (VO2 max) as a risk-stratifying triage tool. We conclude that structured exercise programs can be used to maintain physical activity levels and prevent deconditioning and that VO2 max has the potential to be used as a clinically relevant triage tool during the COVID-19 outbreak." The next article maintains that physical inactivity complicates the severity of COVID-19. It discusses the interaction between physical activity and COVID-19 and maintains the importance of continued and possibly increased physical activity. It voices the concern that the COVID-19 pandemic may further erode physical activity behavior. The following excerpt provides further details:

\section{“G. Hall, D. Laddu, S. Phillips, et al. "A Tale of two Pandemics: How will COVID-19 and Global trends in Physical Inactivity and Sedentary behavior Affect One Another?" https://doi.org/10.1016/j.pcad.2020.04.005}

The world is experiencing an extraordinary, life-altering challenge due to the COVID-19 pandemic [2]. While of a different nature, the world has been living with another pandemic for several years - physical inactivity (PI) and Sedentary Behavior (SB) [13]. According to the World Health Organization, 31\% of individuals 15 years or older are physically inactive and approximately 3.2 million deaths per year are attributed to this unhealthy lifestyle behavior [14]. Previous research has assessed the lasting impact of natural disasters on PA. Following the 2011 earthquake and tsunami that devastated East Japan, Okazaki et al. [15] reported a lasting significant decrease in PA in children and adolescents over three years following the disaster. Similar research must be conducted after we recover from the COVID-19 pandemic to determine the lasting impact this global crisis may have on PA patterns and sedentary time. There are efforts to help individuals be physically active during COVID-19 that should be applauded. The American College of Sports Medicine has released information on how to remain active during COVID-19) [16]. Numerous fitness centers have also been posting free online workout routines to help people remain active at home) [17]. Leading health and wellness journalists have also been stressing the importance of continuing to move during COVID-19) [18] noting recent research that taking as little as 4000 steps per day at any pace, which you can do around your house, significantly improves long-term health ) [19]. Lastly, world renowned exercise and physical activity scholars are also beginning to publish on this topic [20]. While these are certainly positive that must continue, concern remains that individuals who were not previously engaged in a regular exercise routine and led a sedentary lifestyle will not be likely to increase their daily PA during COVID-19 and, in fact, may be moving even less. To this point, we have discussed the potential detrimental impacts of the COVID-19 pandemic on PA behaviors and SB. It is important to note that sedentary behaviors may worsen the impact of future 
pandemics. Not surprisingly, individuals infected with COVID-19 are much more likely to be hospitalized and have poorer health outcomes if underlying medical conditions, such as one or more chronic disease diagnoses, are present [21]. Moreover, the evidence linking a significantly higher increased risk for chronic disease if you are physically inactive and lead a sedentary lifestyle is beyond dispute [22]. The intersection between current risks for health complications and mortality rates associated with COVID-19 and the current state of PI and SB cannot be ignored. If the prevalence of chronic conditions brought about by unhealthy lifestyles were lower, would the catastrophic effects of the COVID-19 pandemic be lessened?"

The next article discuss how physical activity has consistently shown to reduce the risk of systemic inflammation and to improve the immune system. The following excerpt provides additional information about the need for exercise prescription:

\section{“D. Laddu, C. Lavie, S. Phillips, et al. "Physical activity for Immunity Protection: Inoculating Populations with Healthy Living Medicine in Preparation for the Next Pandemic." https://doi.org/10.1016/j. pcad.2020.04.006}

Physical activity (PA) represents one of the primary pillars of health living and is thus a primary component of healthy living medicine [23]; PA is consistently shown to dramatically reduce the risk for developing systemic inflammation, excess body mass and non-communicable diseases known to compromise immune function. [24]. In the context of the novel coronavirus outbreak, questions regarding the potential role of PA as an immune function adjuvant to reduce risk of communicable disease (e.g., bacterial and viral infections) have increased appreciably. The consensus across the exercise immunology literature suggests that the immune system is responsive to exercise, however adaptations to immune system responses to exercise depend on the intensity and duration of effort and type of exercise [25]. Following the 2009 H1N1 influenza epidemic, mounting epidemiological evidence has demonstrated a dose response relationship between PA performed before infection and a reduction in the incidence, duration, or severity of selfreported [26] and laboratory or hospital adjudicated [27] acute upper respiratory tract infections (URTI). Additional investigations have noted that the lower rate of URTI (weighted mean reduction, $\sim 28 \%$ ) is associated with regular engagement in moderate-to-high PA levels as compared to lower PA levels [28] Significant reductions in mortality risk attributed to respiratory disease, pneumonia, and aspiration pneumonia has also been reported in runners, including those with diabetes [29].

While additional high-quality studies are needed to confirm the robust effectiveness of exercise in altering infection prognosis [30], more consistent evidence from randomized clinical trials in adults have shown appreciable reductions in the symptom days during an infectious episode [26]. Appropriately, a growing body of research from experimental studies and animal models have aimed to elucidate the potential mechanism of action underlying the protective effect of moderate-intensity PA against viral respiratory infections. Given the known beneficial effects of habitual exercise on aspects of immunity in younger populations, PA is suggested to be a logical therapeutic strategy to moderate the effects of aging on the immune system and counteract the detrimental effects of reduced immunity due to aging. This contention is well supported by a growing body of evidence from epidemiological and experimental and studies in older adults indicating that regular participation in moderate-intensity exercise attenuates age-related oxidative stress and reduces the frequency of various immune biomarkers that are associated with compromised immunity, thereby suggesting that exercise may delay the onset of immunity reduction due to aging and attenuate the risk of infection [31].

Building on this hypothesis, older adults aged 66-84 years who regularly engaged in PA were observed to have a lower incidence of URTI and a reduced duration of infectious days at an annual followup. The major key points described in this commentary expand on the benefits of PA with well supported evidence demonstrating the potency of regular PA in enhancing immune function and reducing the risk, duration or severity of viral infections. The most consistent evidence suggests routine participation ( 150 min per week) of moderate-intensity physical is necessary to achieve optimal immune support. However, even acute bouts of PA have shown to provide protection from viral infections, therefore supporting the notion that just moving more in the form of structured activity each day may be an important strategy for optimizing the functional integrity of the immune system to prevent or attenuate severity of infection, especially among vulnerable populations with immune compromised conditions."

The following excerpt discusses how exercise can improve angiotensin converting enzyme-2 (ACE2) and how it acts as a therapeutic strategy against COVID-19:

\section{“K.S. Heffernan, S. Young Jae, Exercise as Medicine for COVID-19: an ACE in the Hole?, Medical Hypotheses (2020), doi: https://doi.org/10.1016/j. mehy.2020.109835}

The COVID-19 pandemic is currently exacerbating another established global pandemic - physical inactivity. [32]. The World Health Organization attributes approximately 3.2 million deaths per year to sedentary behavior. For many, social distancing and quarantine coupled with the systemic closure of fitness centers and public parks have imposed unique structural barriers to maintaining a physically active lifestyle. From a public health perspective, the importance of not conflating shelter-in-place with staying-in-place needs to be reinforced. Herein, exercise will be discussed as a possible therapeutic strategy to bolster resilience against COVID-19 via effects on ACE2. Since the angiotensin converting enzyme-2 (ACE2) receptor has been acknowledged 
as an important cellular entry point for SARS-CoV-2, controversy has ensued regarding use of angiotensin-converting enzyme inhibitors (ACEi) and angiotensin II receptor blockers (ARBs) in the management of COVID-19 patients with hypertension. Given the inherent limitations of a retrospective cohort study, care should be taken with interpretation of findings. Nonetheless, results are provocative and shine a light on the complexity of ACE2 in COVID-19 pathophysiology.

The question arises - what can be done to maintain or restore the natural balance between the ACE2-Ang1-7-Mas receptor axis and the ACE-Ang II-AT1 receptor pathway as a possible means of mitigating COVID-19 susceptibility and subsequent risk upon exposure? [33] In one word - exercise. Exercise training can augment the ACE2-Ang1-7-Mas receptor axis while simultaneously inhibiting the ACE-Ang II-AT1 receptor pathway [34]. At a time when many individuals are choosing to move less, the message that exercise is medicine is needed more. Exercise may be an ACE in the hole to help lower risk of COVID-19 infection and minimize the cardiopulmonary sequel during recovery." The excerpts from the next article discusses the importance of physical activity to reduce predisposed conditions and to increase the presence of ACE2, the anti-inflammatory angiotensin converting enzyme 2 :

\section{"C. Kenyon. "The Forrest Gump Approach to Preventing Severe COVID-19 e Reverse the Predisposing Pro- InflammatoryStatewith Exercise".MicrobesandInfection, https://doi.org/10.1016/j.micinf.2020.05.003}

Studies show that the vast majority of those who get severe disease have predisposing conditions such as hypertension, obesity, cardiovascular disease, diabetes or older age [35-38]. For example, in New York, of the 5700 COVID-19 patients sick enough to need hospitalization, $57 \%$ had high blood pressure, $42 \%$ were obese and 34\% had diabetes [35]. Why should hypertension and cardiovascular disease be such prominent risk factors for severe disease in a viral infection? Increasing evidence suggests that the reason why only certain chronic diseases predispose to severe disease is that they are associated with a pro-inflammatory state and an imbalance between the pro-inflammatory angiotensin converting enzyme-1 (ACE1) and anti-inflammatory ACE2 axes in particular $[39,40]$. The chronic diseases associated with COVID-19 are typified by a dominance of the ACE1 axis which promotes vasoconstriction as well as inflammatory, oxidative and fibrotic tissue damage $[39,40]$. A sedentary, excess-calorie-lifestyle results in the same proinflammatory ACE1 bias [41]. So does aging without staying fit [42]. This low-grade tissue inflammation may be critical in determining who gets severe COVID-19 [39-40]. These insights suggest that dealing with the current pandemic and preventing future pandemics and other health disasters will ultimately require rebalancing each of these levels [43-45]. Each of us could start this process by donning our running shoes more regularly."

The excerpts from the next article makes physical activity recommendations to reduce the risk of viral infection:

“G.Jurak, S. Morrison, B. Leskosek, M.Kovac, etal. “Physical activity recommendations during the COVID-19 virus outbreak", Journal of Sport and Health Science (2020), doi: https://doi.org/10.1016/j.jshs.2020.05.003.

Physical Activity (PA) significantly reduces the risk of viral infections [46,47] and levels of anxiety [48,49]. In addition to its short-term benefits, regular PA lowers one's risk of suffering from chronic health conditions (e.g., diabetes, coronary heart disease, and hypertension) which are cited as the most frequent comorbidities in recent studies of COVID-19. [50] Because of the COVID-19 pandemic that has become a global problem, many will face the necessity of requiring hundreds of millions of people to volitionally self-confine themselves in their own homes, or even be "locked down" in a quarantined community. Confinement affects most aspects of human physiology considerably. The logistical issues governing all aspects of daily life that hundreds of millions of people are now confronted with during the current COVID-19 outbreak are unprecedented. It is critical that all persons take collective responsibility of their actions as never before.

Avoid inactivity throughout the day and be aware that any activity you do is better than no activity at all. All persons (who are physically able), should try to accumulate at least $60 \mathrm{~min}$ (but preferably more) of low-to-moderate intensity PA each day, but as stated before every minute counts. At least $15 \mathrm{~min}$ of this activity should take place outside, in natural light, whenever possible. If there is no other option due to political, health risk, or other constraints, use a balcony or exercise by open window. Some activities should be of longer duration (e.g., walking or cycling outside for $30 \mathrm{~min}$ ), and other activities should incorporate strength and flexibility, ideally performed with family members. Finally, exercise, sports medicine, and public health professionals, public health organizations, and national and local public health agencies should all work together to facilitate promotion of safe PA during this public health emergency crisis in which many are infected with COVID-19 but many more affected by the life in quarantine which poses a challenge to maintaining physical and mental well-being."

The following excerpts from the final article recommends high volume, low to medium intensity physical activity to reduce the increased risks of COVID-19 due to isolation and increased sedentary behavior: 
"Marco Narici, Giuseppe De Vito, Martino Franchi, Antonio Paoli, et al. "Impact of Sedentarism Due to the COVID-19 Home Confinement on Neuromuscular, Cardiovascular and Metabolic Health: Physiological and Pathophysiological Implications and Recommendations for Physical and Nutritional Countermeasures." (2020) Eur J Sport Sci. May 12;1-22. doi: 10.1080/17461391.2020.1761076. Online ahead of print.

The COVID-19 pandemic is an unprecedented health crisis as entire populations have been asked to self-isolate and live in homeconfinement for several weeks to months, which in itself represents a physiological challenge with significant health risks. This paper describes the impact of sedentarism on the human body at the level of the muscular, cardiovascular, metabolic, endocrine and nervous systems and is based on evidence from several models of inactivity, including bed rest, unilateral limb suspension, and stepreduction. Data form these studies show that muscle wasting occurs rapidly, being detectable within two days of inactivity. This loss of muscle mass is associated with fiber denervation, neuromuscular junction damage and upregulation of protein breakdown, but is mostly explained by the suppression of muscle protein synthesis. Inactivity also affects glucose homeostasis as just few days of step reduction or bed rest, reduce insulin sensitivity, principally in muscle. Additionally, aerobic capacity is impaired at all levels of the 02 cascade, from the cardiovascular system, including peripheral circulation, to skeletal muscle oxidative function. Positive energy balance during physical inactivity is associated with fat deposition, associated with systemic inflammation and activation of antioxidant defenses, exacerbating muscle loss. Importantly, these deleterious effects of inactivity can be diminished by routine exercise practice, but the exercise dose-response relationship is currently unknown. Nevertheless, low to medium-intensity high volume resistive exercise, easily implementable in home-settings, will have positive effects, particularly if combined with a $15-25 \%$ reduction in daily energy intake. This combined regimen seems ideal for preserving neuromuscular, metabolic and cardiovascular health."

After reviewing the current literature regarding physical activity and COVID-19 it is apparent that physical activity may well be a major factor that reduces the severe pathologies resulting from the infection of COVID-19. If people are dictated or choose to remain at home it is essential not to "veg out" and become a "couch potato." Sheltering-in-place confinement strategies and physical activity are not mutually exclusive. If sheltered in place then it is necessary to find ways to stay physically active, adjust caloric intake to reflect current activity levels, and to reduce sedentary lifestyles. Not only will this type of behavior reduce the risk of detrimental health effects from COVID-19, it will also improve general health.

\section{References}

1. Nelson K, Williams C, Graham N (2005) Infectious Disease Epidemiology Theory and Practice. Jones and Bartlett Publishers: Chapter 2: 17-27; Chapter 3: 50-54.

2. (2020) World Health Organization. Coronavirus disease (COVID-19) Pandemic.

3. Halliwell B, Gutteridge J (1984) Oxygen toxicity, oxygen radicals, transition metals and disease. Biochem J 219: 1-14.

4. Zelko I, Mariani T, Folz R (2002) Superoxide dismutase multigene family: a comparison of the CuZn-SOD (SOD1), Mn-SOD (SOD2), and EC-SOD (SOD3) gene structures, evolution, and expression. Free Radic Biol Med 33: 337-349.

5. Forrester S, Kikuchi D, Marina S. Hernandes, Qian Xu, Kathy K Griendling (2018) Reactive oxygen species in metabolic and inflammatory signaling. Circ Res 122: 877-902.

6. Folz R, Guan J, Seldin M, TD Oury, J J Enghild, et al. (1997) Mouse extracellular superoxide dismutase: primary structure, tissuespecific gene expression, chromosomal localization, and lung in situ hybridization. Am J Respir Cell Mol Biol 17: 393-403.

7. Chen Y, Hou M, Li Y, Jay H Traverse, Ping Zhang, et al. (2005) Increased superoxide production causes coronary endothelial dysfunction and depressed oxygen consumption in the failing heart. Am J Physiol Heart Circ Physiol 288: H133-H141.

8. Fattman C, Schaefer L, Oury T (2003) Extracellular superoxide dismutase in biology and medicine. Free Radic Biol Med 35: 236-256.

9. Arcaroli J, Hokanson J, Abraham E, Mark Geraci, James R Murphy, et al. (2009) Extracellular superoxide dismutase haplotypes are associated with acute lung injury and mortality. Am J Resp Crit Care Med 179: 105112 .

10. De Sousa C, Sales M, Rosa T, John Eugene Lewis 3, Rosangela Vieira de Andrade, et al. (2017) The antioxidant effect of exercise: a systematic review and meta-analysis. Sports Med 47: 277-293.

11. Call J, Chain K, Martin K, Vitor A Lira, Mitsuharu Okutsu, et al. (2015) Enhanced skeletal muscle expression of extracellular superoxide dismutase mitigates streptozotocin-induced diabetic cardiomyopathy by reducing oxidative stress and aberrant cell signaling. Circ Heart Fail 8: 188-197.

12. Wadley A, Keane G, Cullen T, Lynsey James, Jordan Vautrinot, et al. (1985) Characterization of extracellular redox enzyme concentrations in response to exercise in humans. J Appl Physiol 127: 858-866.

13. Pratt M, Ramirez V, Salvo D, Harold W Kohl Iii, Ding Ding, et al. (2019) Attacking the pandemic of physical inactivity: what is holding us back? British Journal of Sports Medicine. bjsports-2019-101392.

14. Ding D, Lawson K, Kolbe-Alexander T, Eric A Finkelstein 4, Peter T Katzmarzyk, et al. (2016) The economic burden of physical inactivity: a global analysis of major non-communicable diseases. The Lancet 388(10051): 1311-1324

15. Okazaki K, Suzuki K, Sakamoto Y, Keiji Sasaki (2015) Physical activity and sedentary behavior among children and adolescents living in an area affected by the 2011 Great East Japan earthquake and tsunami for 3 years. Prev Med Rep 2:720-724.

16. (2020) American College of Sports Medicine. Staying Active During the Coronavirus Pandemic.

17. Wallace A (2020) People welcome online workouts to fill gap left by shuttered gyms and studios. Washington Post.

18. Reynolds G (2020) Stuck Inside? Keep Walking. NY Times. 
19. Saint-Maurice P, Troiano R, Bassett Jr D, Barry I Graubard , Susan A Carlson ,et al. (2020) Association of daily step count and step intensity with mortality among US adults. Jama 323(12): 1151-1160.

20. Jimenez-Pavon D, Carbonell-Baeza A, Lavie C (2020) Physical exercise as therapy to fight against the mental and physical consequences of COVID-19 quarantine: special focus in older people. Prog Cardiovasc Dis.

21. Chow N, Fleming-Dutra K, Gierke R, et al. (2020) Preliminary estimates of the prevalence of selected underlying health conditions among patients with Coronavirus disease 2019- United States, February 12-March 28, 2020.Morbidity and mortality weekly report: 69(13).

22. Bauer U, Briss P, Goodman R, Barbara A Bowman (2014) Prevention of chronic disease in the 21st century: elimination of the leading preventable causes of premature death and disability in the USA. Lancet (London, England) 384(9937): 45-52.

23. Arena R, McNeil A, Sagner M, Carl J Lavie (2017) Healthy living: the universal and timeless medicine for healthspan. Prog Cardiovasc Dis 59: 419-421.

24. Fletcher G, Landolfo C, Niebauer C, Cemal Ozemek, Ross Arena, et al. (2018) Promoting physicalactivity and exercise: JACC health promotion series. J Am Coll Cardiol 72: 1622-1639.

25. Nieman D, Wentz L (2019) The compelling link between physical activity and the body's defense system. J Sport Health Sci 8: 201-217.

26. Nieman D, Henson D, Austin M, Wei Sha (2011) Upper respiratory tract infection is reduced in physically fit and active adults. Br J Sports Med 45: 987-992.

27. Siu E, Campitelli M, Kwong J (2012) Physical activity and influenza-coded outpatient visits, a population-based cohort study. PloS one 7: e39518.

28. Fondell E, Lagerros Y, Sundberg C, Mats Lekander, Olle Bälter, et al. (2011) Physical activity, stress, and self-reported upper respiratory tract infection. Med Sci Sports Exerc 43: 272-279.

29. Lavie C, Lee D, Sui X, Ross Arena 4, James H O'Keefe, et al. (2015) Effects of running on chronic diseases and cardiovascular and all-cause mortality. Mayo Clin Proc 90: 1541-1552.

30. Grande A, Keogh J, Hoffmann T, et al. (2015) Exercise versus no exercise for the occurrence, severity and duration of acute respiratory infections. The Cochrane database of systematic reviews: 1-54. Cd010596.

31. Simpson R, Lowder T, Spielmann G, Austin B Bigley, Emily C LaVoy, et al. (2012) Exercise and the aging immune system. Ageing Res Rev 11: 404-420.

32. Hall G, Laddu D, Phillips S, Carl J Lavie, Ross Arena (2020) A tale of two pandemics: How will COVID-19 and global trends in physical inactivity and sedentary behavior affect one another? Progress in Cardiovascular Diseases (2020).

33. Yan T, Xiao R, Lin G (2020) Angiotensin-converting enzyme 2 in severe acute respiratory syndrome coronavirus and SARS-CoV-2: A doubleedged sword? FASEB journal: official publication of the Federation of American Societies for Experimental Biology (2020).

34. Nunes-Silva A, Rocha G, Magalhaes D, Lucas Neves Vaz, Marcelo Henrique Salviano de Faria, et al. (2017) Physical Exercise and ACE2-Angiotensin(1-7)-Mas Receptor Axis of the Renin Angiotensin System. Protein and peptide letters 24: 809-816.
35. Richardson S, Hirsch J, Narasimhan M, James M. Crawford, Thomas McGinn, et al. (2020) Presenting characteristics, comorbidities, and outcomes among 5700 patients hospitalized with COVID-19 in the New York City area. J Am Med Assoc 2020.

36. Onder G, Rezza G, Brusaferro S (2020) Case-fatality rate and characteristics of patients dying in relation to COVID-19 in Italy. J Am Med Assoc 2020.

37. Wu Z, McGoogan M (2020) Characteristics of and important lessons from the coronavirus disease 2019 (COVID-19) outbreak in China: summary of a report of 72,314 cases from the Chinese Center for Disease Control and Prevention. J Am Med Assoc 323: 1239e42.

38. Mehra M, Desai S, Kuy S, Timothy D. Henry, Amit N Patel (2020) Cardiovascular disease, drug therapy, and mortality in COVID-19. N Engl J Med 2020.

39. Henry B, Vikse J, Benoit S, Emmanuel J Favaloro, Giuseppe Lippi (2020) Hyperinflammation and derangement of Renin-AngiotensinAldosterone System in COVID-19: a novel hypothesis for clinically suspected hypercoagulopathy and microvascular immunothrombosis. Clin Chim Acta 507: 167e73.

40. Sriram K, Insel P (2020) A hypothesis for pathobiology and treatment of COVID-19: the centrality of ACE1/ACE2 imbalance. Br J Pharmacol 2020.

41. Migdalis I, Iliopoulou V, Kalogeropoulou K, K Koutoulidis, M Samartzis (1990) Elevated serum levels of angiotensin-converting enzyme in patients with diabetic retinopathy. South Med J 83: 425e7.

42. Ferrucci L, Fabbri E (2018) Inflammageing: chronic inflammation in ageing, cardiovascular disease, and frailty. Nat Rev Cardiol 15: 505e22.

43. Dunk J, Jones D, Capon A (2019) Human health on an ailing planet -historical perspectives on our future. N Engl J Med 381:778e82.

44. Keesing F, Belden L, Daszak P, C Drew Harvell, Robert D Holt, et al. (2010) Impacts of biodiversity on the emergence and transmission of infectious diseases. Nature 468: 647e52.

45. Ahmed F, Ahmed N, Pissarides C, Joseph Stiglitz (2020) Why inequality could spread COVID-19. Lancet Pub Health (2020).

46. Martin S, Pence B, Woods J (2009) Exercise and respiratory tract viral infections. Exerc Sport Sci Rev 37: 157-164.

47. Romeo J, Wärnberg J, Pozo T, A Marcos (2010) Physical activity, immunity and infection. Proc Nutr Soc 69: 390-399.

48. Anderson E, Shivakumar G (2013) Effects of exercise and physical activity on anxiety. Front Psychiatry 4: 27.

49. Rebar A, Stanton R, Geard D, Camille Short, Mitch J Duncan, et al. (2015) A meta-meta-analysis of the effect of physical activity on depression and anxiety in non-clinical adult populations. Health Psychol Rev 9: 366-378.

50. Fang L, Karakiulakis G, Roth M (2020) Are patients with hypertension and diabetes mellitus at increased risk for COVID-19 infection? Lancet Resp Med 8: e21. 
ISSN: 2574-1241

DOI: $10.26717 /$ BJSTR.2020.29.004733

Marc Bonis. Biomed J Sci \& Tech Res

(c) (P) This work is licensed under Creative Commons Attribution 4.0 License

Submission Link: https://biomedres.us/submit-manuscript.php

$\begin{array}{ll}\text { BIOMEDICAL } & \text { Assets of Publishing with us } \\ \text { RESEARCHES } & \text { - Global archiving of articles } \\ \text { - Immediate, unrestricted online access } \\ \text { - Rigorous Peer Review Process }\end{array}$

